\title{
Digital Alloys of AIN/AIGaN for Deep UV Light Emitting Diodes
}

\author{
Sergey A. Nikishin*, Mark Holtz and Henryk Temkin \\ Nano Tech Center, Texas Tech University, Lubbock, TX 79409, U.S.A. \\ (Received February 14, 2005; accepted April 27, 2005; published October 11, 2005)
}

We report a systematic study of the optical and electrical properties of deep ultraviolet light emitting diodes based on digital alloy structures of $\mathrm{AlN} / \mathrm{Al}_{0.08} \mathrm{Ga}_{0.92} \mathrm{~N}$ grown by gas source molecular beam epitaxy with ammonia. Digital alloys are formed by short period superlattices consisting of $\mathrm{Al}_{0.08} \mathrm{Ga}_{0.92} \mathrm{~N}$ wells, 0.50 or $0.75 \mathrm{~nm}$ thick, and $\mathrm{AlN}$ barriers, 0.75 to $1.5 \mathrm{~nm}$ thick. For digital alloys with effective bandgap of $5.1 \mathrm{eV}$, average AlN composition $72 \%$, we obtain room temperature electron concentrations up to $1 \times 10^{19} \mathrm{~cm}^{-3}$ and resistivity of $0.005 \Omega \cdot \mathrm{cm}$ and hole concentrations of $1 \times 10^{18} \mathrm{~cm}^{-3}$ with resistivity of $6 \Omega \cdot \mathrm{cm}$. Light emitting diodes based on digital alloys are demonstrated operating in the range of 250 to $290 \mathrm{~nm}$.

[DOI: 10.1143/JJAP.44.7221]

KEYWORDS: LED, GSMBE, ultraviolet, AIGaN, short period superlattice, digital alloys

\section{Introduction}

The large bandgap $\mathrm{Al}_{x} \mathrm{Ga}_{1-x} \mathrm{~N}$ alloys are the semiconductors of choice for deep ultraviolet (UV) light emitting diodes (LEDs) operating below $300 \mathrm{~nm} .{ }^{1-7)}$ There are many emerging applications for UV LEDs in spectroscopy, chemical and biological analysis, and water and food treatment. ${ }^{8-11)}$ LEDs are preferred for these applications because they are small, power efficient, contain no hazardous materials, are highly resistant to shock, and have very long lifetimes. High power UV LEDs are considered as replacements for conventional light sources for many industrial and commercial purposes, at a potentially enormous world-wide energy savings.

$\mathrm{Al}_{x} \mathrm{Ga}_{1-x} \mathrm{~N}$ alloys can be grown epitaxially in the wurtzite structure across the full composition range using several methods, including gas source molecular beam epitaxy (GSMBE) with ammonia. The energy gap smoothly increases from 3.4 in $\mathrm{GaN}$ to $6.2 \mathrm{eV}$ in $\mathrm{AlN}$, providing a wide range of possible emission wavelengths. However, device preparation is fundamentally limited by the difficulty of preparing p-type layers of large bandgap $\mathrm{Al}_{x} \mathrm{Ga}_{1-x} \mathrm{~N}$. We describe here how this limitation can be overcome, and to what extent, by the use of digital alloy structures (DAS) formed by short period superlattices (SLs) consisting of 3-6 monolayer (ML) thick barriers of AlN and 2-3 ML thick wells of $\mathrm{Al}_{x} \mathrm{Ga}_{1-x} \mathrm{~N} .{ }^{1,4,6,12)}$ The average DAS composition and therefore the effective energy gaps of $\mathrm{AlN} / \mathrm{AlGaN}^{13}$ ) and $\mathrm{AlN} / \mathrm{GaN}^{14,15)}$ SLs are known to depend on the well/ barrier thickness ratio, the well composition, and the SL period. We have shown that DAS containing as many as 400 well-barrier pairs can be reproducibly grown by GSMBE with excellent structural and optical properties. ${ }^{1,4,6)}$ When doped with $\mathrm{Mg}$, room temperature hole concentrations of $1 \times 10^{18} \mathrm{~cm}^{-3}$ were obtained in DAS with an average AlN content of $\sim 65 \% .^{4,6,16)}$ In similar DAS doped with $\mathrm{Si}$, electron concentrations of $3 \times 10^{19} \mathrm{~cm}^{-3}$, were reached with room temperature mobility of $10-20 \mathrm{~cm}^{2} /(\mathrm{V} \cdot \mathrm{s}) .^{4,6)}$ Based on n- and p-type DAS of $\mathrm{AlN} / \mathrm{Al}_{0.08} \mathrm{Ga}_{0.92} \mathrm{~N}$, we have prepared UV LEDs with peak emission at $280-290 \mathrm{~nm}$ and $262 \mathrm{~nm} \cdot{ }^{1,4,6,17)}$ In this paper we discuss the optical and

*E-mail address: Sergey.Nikishin@coe.ttu.edu electrical properties of the p- and n-type DAS with effective bandgap up to $5.3 \mathrm{eV}(234 \mathrm{~nm})$ and describe the preparation of LEDs operating between 290 to $250 \mathrm{~nm}$.

\section{Growth and Properties of DAS}

Most samples discussed here were grown by GSMBE with ammonia on c-plane sapphire using thin AlN buffer layers. Some structures were grown directly on sapphire, in order to compare their structural, electrical, and optical properties to standard DAS and a number of LED structures were grown on $\mathrm{Si}(111)$ substrates. The DAS samples were either not intentionally doped or doped with Si derived from silane (n-type), or $\mathrm{Mg}$ derived from a conventional effusion cell (p-type). Nominal well thicknesses of $\sim 0.50$ and $\sim 0.75 \mathrm{~nm}$, i.e., 2 and $3 \mathrm{MLs}$, were sandwiched between barrier layers with thickness varying from $\sim 0.5$ to $\sim 1.5 \mathrm{~nm}$, resulting in the range of periods from 1.25 to $2.25 \mathrm{~nm}$.

Reflection high energy electron diffraction (RHEED) was used for in situ monitoring of the growth mode. For DAS grown directly on sapphire, two dimensional (2D) growth mode was confirmed by RHEED pattern $(1 \times 1)$ observed from the onset to the end of epitaxy at high temperature. At low temperature, RHEED shows $(1 \times 1) \rightarrow(3 \times 3)$ and $(1 \times 1) \rightarrow(6 \times 6)$ transitions. This corresponds to the formation of inversion domains (IDs) in our DAS. Similar surface structure transitions, observed using RHEED, have been reported by Shubina et al. ${ }^{18)}$ for AlGaN/GaN SLs with IDs. A transmission electron microscope (TEM) image of DAS, grown directly on sapphire, is shown in Fig. 1(a). The TEM shows that this DAS contains a very high density of ID boundaries (IDBs). X-ray diffraction (XRD) data, not shown here, is consistent with high density of dislocations. We found the formation of IDs starts at the sapphire-DAS interface. Incomplete nitridation of sapphire and low lateral growth rate of $\mathrm{Ga}$ - and $\mathrm{N}$-face domains result in vertical propagation of IDs through the entire structure. The DASs with high density of IDs show the highest cathodoluminescence (CL) intensity. It is well known ${ }^{19,20)}$ that IDBs dominate the light emission process in $\mathrm{GaN}$ containing these defects. Emission intensity from domains having Gaface polarity is weaker than from IDBs by more than one order of magnitude. A similar result was reported for MBE grown AlGaN/GaN SLs. ${ }^{18)}$ However, DASs with high 

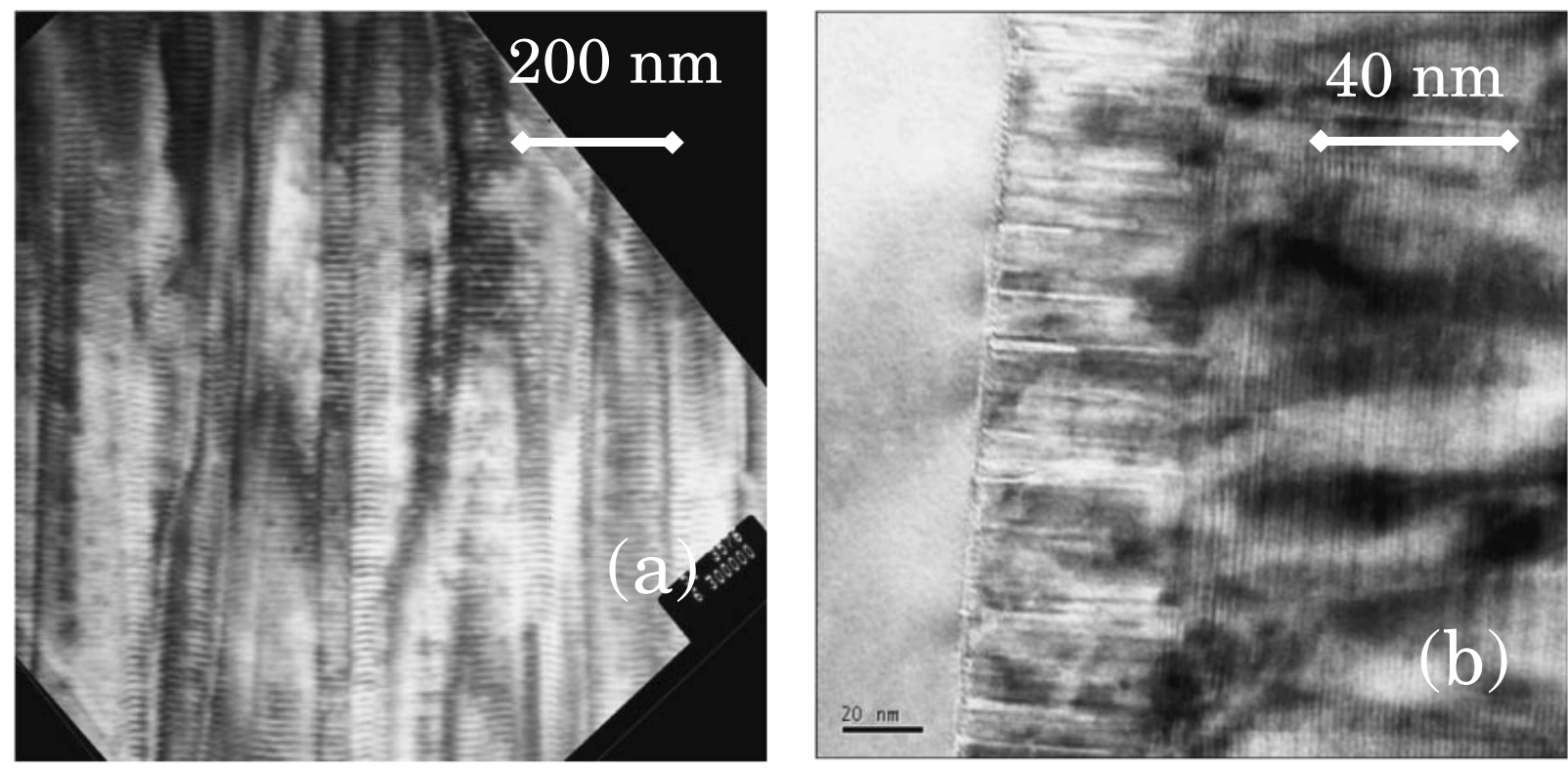

Fig. 1. TEM pictures of digital alloy grown directly on sapphire (a) and on thin AlN buffer (b).

density of IDBs have inferior electrical properties and cannot be used in the preparation of devices.

A thin AlN buffer layer was used as a growth template for most DAS. The process was started by exposing the surface of sapphire to ammonia for $15 \mathrm{~min}$. Following this, the surface was exposed to the flux of $\mathrm{Al}$, for $\sim 3 \mathrm{~s}$, followed by $\sim 3$ s long exposure to ammonia (with the Al flux off). This alternating exposure process, repeated 5 to 10 times, facilitates formation of oriented AlN islands on sapphire. The growth of AlN was then started by turning on ammonia and $\mathrm{Al}$ together. Spots observed at this point in the RHEED pattern indicated formation of oriented three-dimensional (3D) islands of AlN. The formation of the $(1 \times 1)$ surface structure could be seen by RHEED after depositing 5 to $7 \mathrm{~nm}$ of AlN. A completed 2D growth mode could be seen, by RHEED, after about $40 \mathrm{~nm}$. The growth of DAS was started after the AlN buffer layer completed the transition to the $2 \mathrm{D}$ growth mode. Formation of a $(2 \times 2)$ surface structure could be seen after deposition of 20 to $50 \mathrm{~nm}$ of DAS. The $(1 \times 1) \rightarrow(2 \times 2)$ transition corresponds to the formation of a single domain DAS with group-III face polarity. The rapid transition to 2D growth is of interest for two reasons. First, it results in a decrease in the defect density, as shown in the TEM cross section of Fig. 1(b). Second, the rapid transition is needed to assure crack-free samples. We used these DASs grown on AlN buffer for device fabrication.

High resolution XRD was used for structural characterization of DASs. This method is very sensitive to any changes in average composition and period of the DAS. XRD spectra of two DASs, denoted $\mathrm{SL}_{1}$ and $\mathrm{SL}_{2}$, grown one after another on the same substrate are shown in Fig. 2. Details of the 0th order peak are shown in the inset. The average composition of these DASs was determined from the 0th order XRD peak using Vegard's law. The period was determined from the angular positions of the +1 and -1 SL peaks. The presence of these features confirms high degree of periodicity and general structural perfection. Well compositions are determined based on previous growth

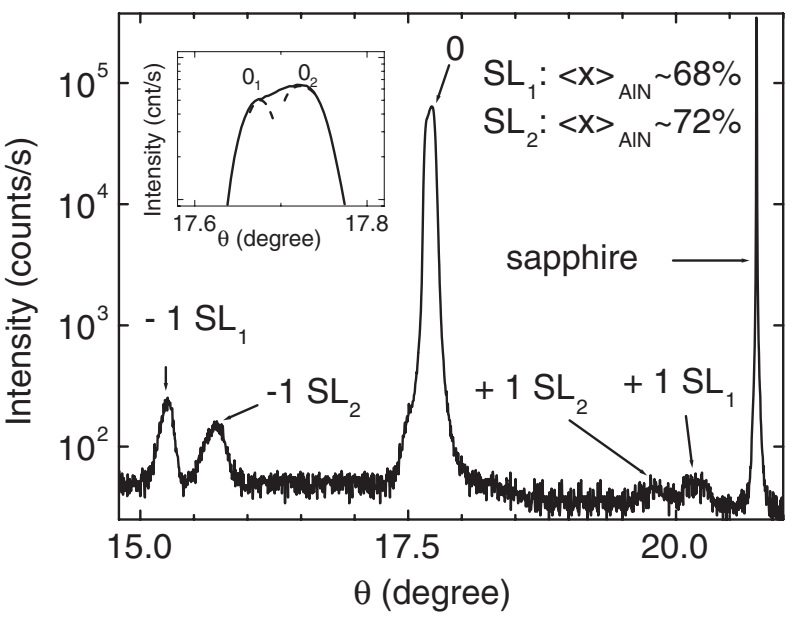

Fig. 2. XRD plot from two DASs grown one after the other. The inset shows detailed features of the 0th order diffraction peaks denoted $\mathrm{SL}_{1}$ and $\mathrm{SL}_{2}$.

experiments of thick layers of the well material under identical GSMBE conditions. Thus, we determine the well and the barrier thicknesses from the measurement of the average composition of the SL and its period. Both SLs in Fig. 2 contain AlN barriers and three ML thick $\mathrm{Al}_{0.08} \mathrm{Ga}_{0.92} \mathrm{~N}$ wells. The number of barrier/well pairs is the same in both SLs. The growth time of AlN barrier in $\mathrm{SL}_{2}$ was increased in order to increase barrier thickness by two MLs. From Fig. 2 we obtain a period $20.15 \pm 0.25 \AA$ for $\mathrm{SL}_{1}$ and a period $25.92 \pm 0.25 \AA$ for $\mathrm{SL}_{2}$. The results are in excellent agreement with TEM data, not shown here. The average composition for $\mathrm{SL}_{1}$ is $\sim 68 \%$ of $\mathrm{AlN}$ and for $\mathrm{SL}_{2}$ it is $\sim 72 \%$ of AlN. This is also in excellent agreement with optical reflectance data, described below, which shows the effective bandgaps of $\mathrm{SL}_{1}$ and $\mathrm{SL}_{2}$ to be 4.9 and $5.1 \mathrm{eV}$, respectively. Detailed dynamic simulations suggest formation of interfacial layers as the main reason for the slight broadening present in the XRD data of these structures. ${ }^{21)}$ 


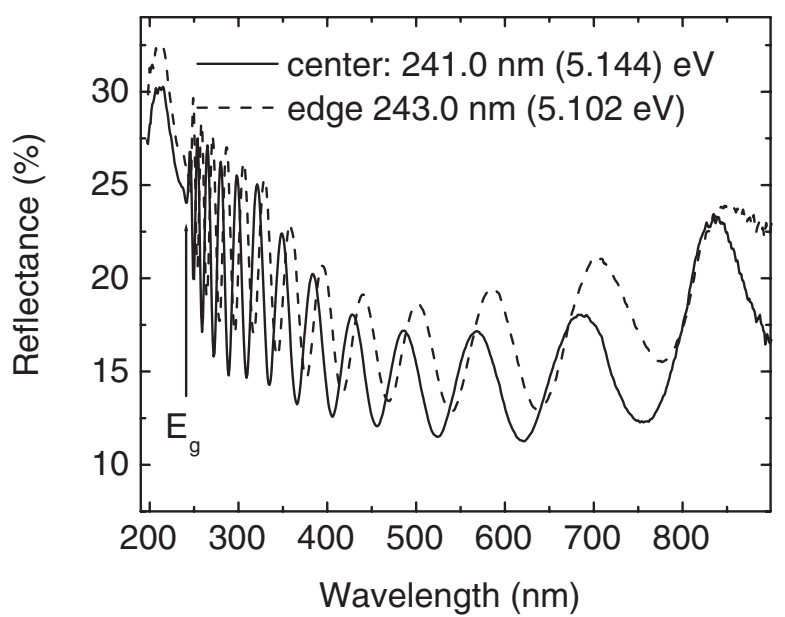

Fig. 3. Optical reflectance spectra obtained from two different positions on one DAS grown on $2^{\prime \prime}$ wafer.

The average composition of DAS depends on the barrier and well growth rates, and the relatively large $\mathrm{Ga}$ and $\mathrm{Al}$ shutter switching times of $\sim 1 \mathrm{~s}$ compared to the $\sim 6 \mathrm{~s}$ well growth time. The interfacial layer can contribute to the optical properties of the DAS by producing an intermediate alloy in the transition region and by resulting in domains with up to one additional monolayer of well or barrier material.

\section{Optical and Electrical Measurements}

Optical properties were studied using optical reflectance and cathodoluminescence (CL). Device structures were also investigated using electroluminescence (EL). Optical reflectance, CL, and EL measurements were carried out with the samples at ambient temperature.

Figure 3 shows typical optical reflectance spectra for a DAS with a very short average period of $1.95 \mathrm{~nm}$ obtained from XRD. The spectrum exhibits Fabry-Perot interference fringes for photon energies below the optical bandgap. Fringe spacing analysis allows determination of the layer thickness. These fringes are attenuated by absorption above the energy gap, allowing us to determine $E_{\mathrm{g}}$ to better than $\pm 50 \mathrm{meV}$ accuracy ${ }^{22)}$ depending on the total layer thickness producing the fringes. Accuracy can be improved further by analyzing the derivative spectrum, which emphasizes the rapid oscillations of the Fabry-Perot fringes and reduces gradual variations in the reflectance. Figure 3 illustrates the typical uniformity of DAS across $2^{\prime \prime}$ wafer. The optical bandgaps varied radially by $\pm 40 \mathrm{meV}$ across the wafer, which is comparable to the uncertainty of the reflectance measurements.

As expected, DAS bandgaps obtained from reflectance measurements increase with the average AlN content. The effective bandgap can be controlled from $\sim 4.5$ to $5.3 \mathrm{eV}$, i.e., from $\sim 276$ to $234 \mathrm{~nm}$, based on the growth parameters. This is illustrated in Fig. 4, where we summarize $E_{\mathrm{g}}$ measured by reflectance vs period of numerous DAS obtained by XRD. Two series of growth experiments are shown, each having DAS with different well thicknesses. The series grown with $3 \mathrm{ML}$ well thickness is denoted "2" while the series with $2 \mathrm{ML}$ well thickness is higher in energy and denoted "1". The open symbols in Fig. 4 are CL peak energies for several of the $3 \mathrm{ML}$ well DAS. Scatter in the

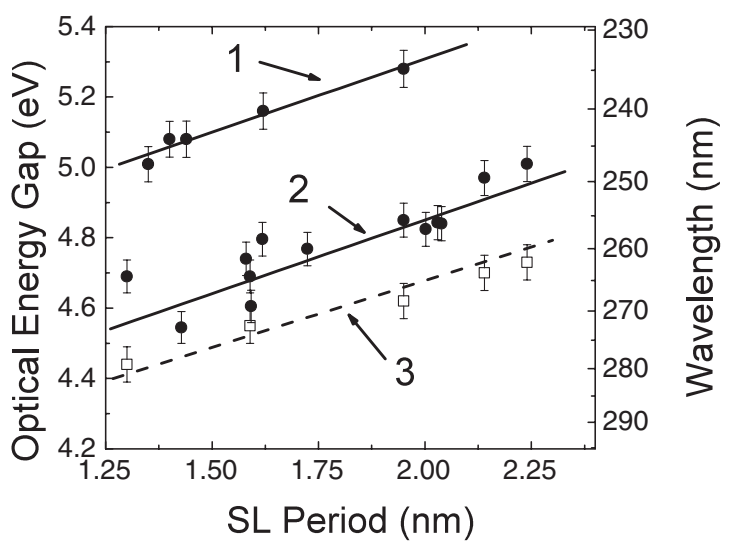

Fig. 4. Summary of optical gaps vs period for DASs grown with two nominal well thicknesses: 2 and $3 \mathrm{ML}$. Solid lines are linear fits to the data. The open symbols are CL peak energies for several DASs. The dashed line is a guide for the eye.

data is attributed mainly to monolayer level uncertainty in the well and barrier widths. Additional contributions are due to local composition fluctuations in $\mathrm{AlGaN}$ well alloy and interfacial barrier/well layer. ${ }^{21)}$ It is clear from our results that control of the DAS well and barrier thickness provides "coarse" and "fine" adjustment of the effective bandgap. By systematically varying the barrier thickness while maintaining constant well thickness a shift $\sim 100 \pm 20 \mathrm{meV} / \mathrm{ML}$ ( $\sim 7 \mathrm{~nm} / \mathrm{ML})$ can be determined. Changing the well thickness with fixed period provides the coarse control of $400 \pm 30 \mathrm{meV}$. Increasing the well by $1 \mathrm{ML}$ and leaving the barrier thickness constant results in a bandgap energy change of $270 \pm 20 \mathrm{meV}$.

CL peak energies for several DAS with $3 \mathrm{ML}$ thick well are shown in Fig. 4 where line " 3 " is a guide to the eye. These data illustrate the typical red shift observed between the emission maximum and the optical bandgap. Several effects can contribute to the observed 200 to $300 \mathrm{meV}$ red shifts in the CL peak relative to the energy gap. Optical reflectance is sensitive to the average effective bandgap of the structure. On the other hand, in emission, the carriers tend to thermalize into the lowest lying states. The Stokes shift, which is related to the excited state configuration, is a factor in all luminescence processes and therefore contributes to the observed red shift. Here, the DAS will form miniband states. The individual wells and barriers in the AlN/ $\mathrm{AlGaN}$ system are also distorted by large piezoelectric fields at interfaces, pushing confined states well below the effective bandedge of the DAS. These factors can result in the large red shift we observe in our DAS. Clearly, more work combining experiments and theory are needed to understand this effect.

All samples were characterized by Hall measurements at temperature varying from 230 to $360 \mathrm{~K}$. Room temperature hole concentrations of $1 \times 10^{18} \mathrm{~cm}^{-3}$ and resistivity of $6 \Omega \cdot \mathrm{cm}$ were measured in DAS with an average AlN content of $\sim 72 \%$. We show in Fig. 5 hole concentrations obtained from room temperature Hall measurements for two different sets of wafers grown under identical conditions while varying the well and barrier growth intervals. For the first set, the $\mathrm{Al}_{0.08} \mathrm{Ga}_{0.92} \mathrm{~N}$ well widths are grown with 7-s duration, and the AlN barrier growth time varied from 15 to 


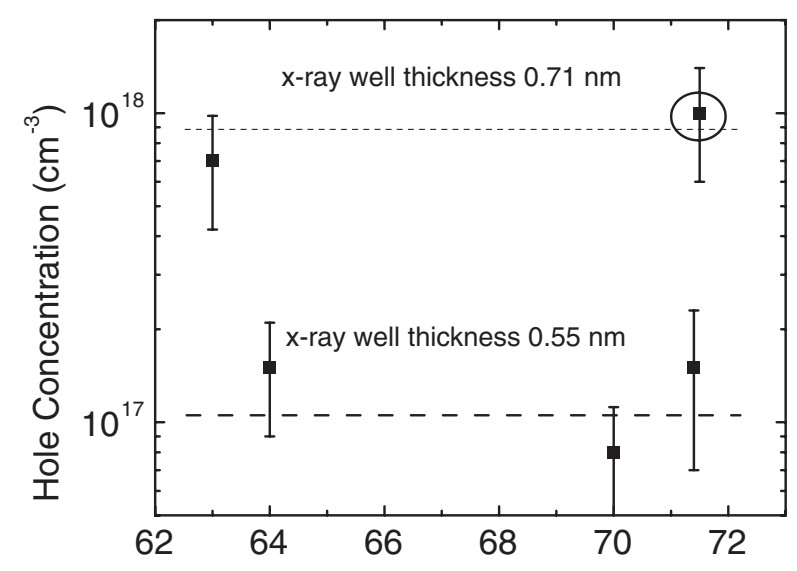

$<x>$ Average AIN Concentration (mole \%)

Fig. 5. Hole concentrations at room temperature from Hall measurements. Temperature dependence of resistivity of circled sample is shown in Fig. 6.

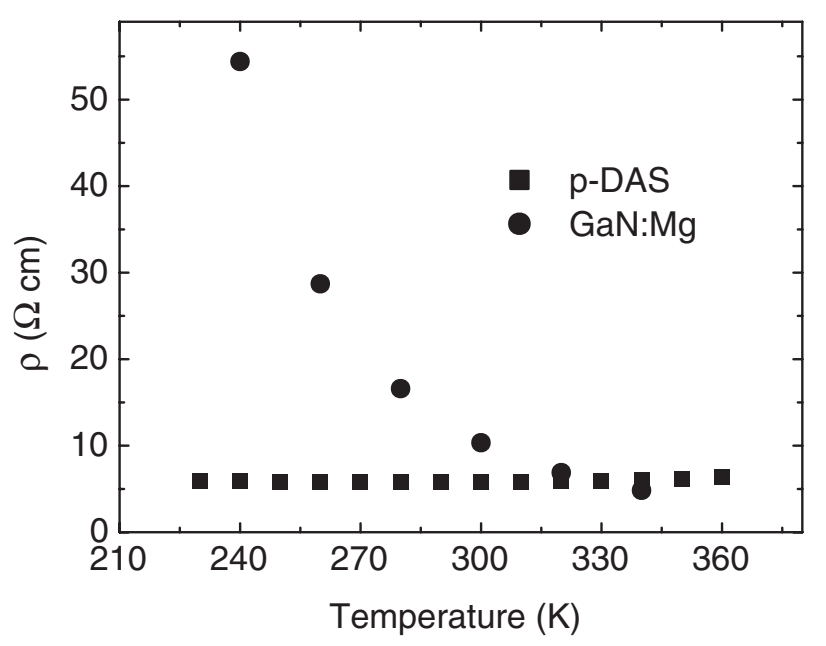

Fig. 6. Temperature dependence of resistivity of p-type digital alloy with average $x_{\mathrm{AlN}} \sim 0.72\left(E_{\mathrm{g}} \sim 5.1 \mathrm{eV}\right)$ and p-type GaN. Hole concentration $1 \times 10^{18} \mathrm{~cm}^{-3}$ in both samples.

$21 \mathrm{~s}$. For the second set, the well widths with the same composition are grown with 12-s duration and the barriers varied as in the previous set. Hole concentrations group near values of $10^{17}$ and $10^{18} \mathrm{~cm}^{-3}$, even though the average well thickness deviates by only one monolayer. Evidently, the hole concentration is very sensitive to well width over the 1.25 to $2.25 \mathrm{~nm}$ range in DAS period studied here. It is clear from this work that acceptable hole concentrations are achievable with an appropriately engineered DAS.

Temperature dependences of resistivity of p-type digital alloy with average $x_{\mathrm{AlN}} \sim 0.72\left(E_{\mathrm{g}} \sim 5.1 \mathrm{eV}\right)$ and p-type $\mathrm{GaN}$ are shown in Fig. 6 . The hole concentration of $1 \times 10^{18}$ $\mathrm{cm}^{-3}$ was measured for both samples at room temperature. Behavior of $\mathrm{GaN}$ resistivity is typical for bulk materials and the resistivity is seen to decrease with increasing temperature. In contrast, for the DAS with very high average AlN composition, $72 \%$, the resistivity is very weakly temperature dependent. This can be attributed to formation of 2D hole gas in our digital alloys. For Si-doped n-type DASs with similar composition, we obtain electron concentration in the

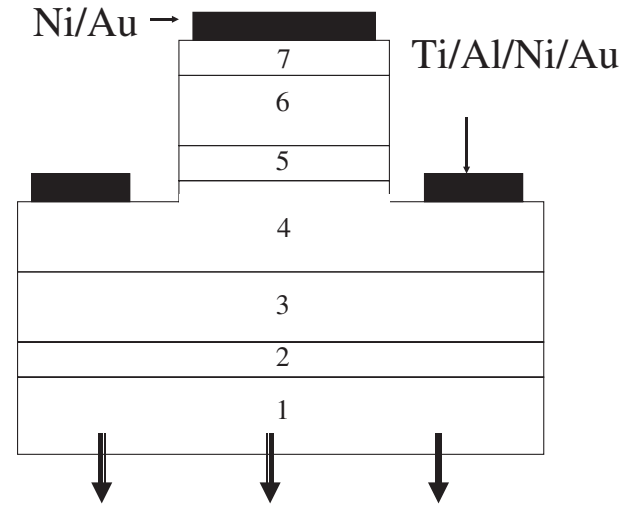

Fig. 7. Schematic cross-section of a $260 \mathrm{~nm}$ LED designed for light emission through the sapphire substrate (1). Layer (2) is a $40 \mathrm{~nm}$ thick AlN buffer layer, (3) n-type $\mathrm{Al}_{0.7} \mathrm{Ga}_{0.3} \mathrm{~N}$ :Si buffer layer, (4) n-type DAS with $x_{\mathrm{AlN}} \sim 0.72,(5)$ undoped DAS active region of $\sim 30 \mathrm{~nm},(6)$ p-type DAS with $x_{\text {AlN }} \sim 0.72$, and (7) $5 \mathrm{~nm}$ thick p-GaN:Mg contact layer.

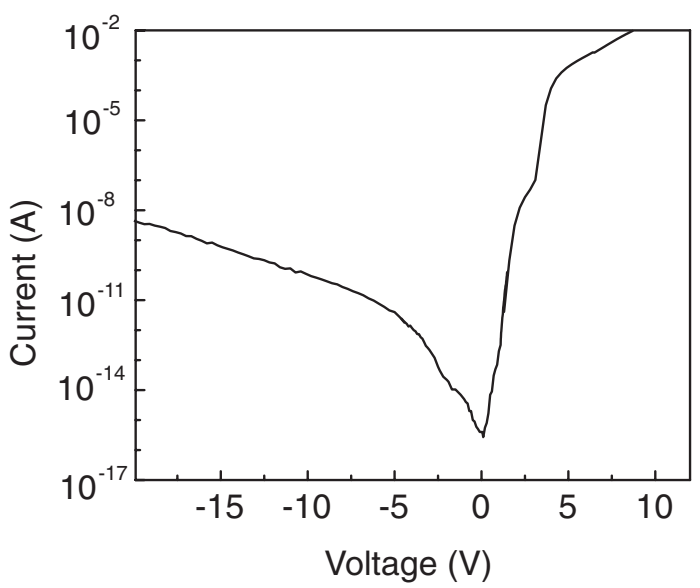

Fig. 8. $I-V$ characteristics of a high-quality LED operating at $260 \mathrm{~nm}$.

range of $\sim 1 \times 10^{19} \mathrm{~cm}^{-3}$ and resistivity of $\sim 0.005 \Omega \cdot \mathrm{cm}$ at room temperature. Likewise, we observe no temperature dependence of the resistivity.

Based on these results we prepared double heterostructure (DH) LEDs operating at different wavelengths. Details of the DH design are described elsewhere. ${ }^{1,4,6)}$ A schematic cross-section of a typical $260 \mathrm{~nm}$ LED is illustrated in Fig. 7. In all LEDs, the $\mathrm{p}$ - and n-type cladding layers were grown with the same effective bandgap corresponding to $x_{\mathrm{AlN}} \sim$ 0.72 . The emission wavelengths were controlled through the effective bandgap of the active region, based on Fig. 4. Mesa structure LEDs were prepared using $\mathrm{Cl}_{2}$ plasma etching, as previously described. ${ }^{23,24)}$ The current-voltage $(I-V)$ characteristics of a representative LED are shown in Fig. 8. The device turns on at $\sim 5.0 \mathrm{~V}$, as expected for a device with active layer bandgap of $\sim 4.8 \mathrm{eV}$. Very low dark leakage currents, $7 \times 10^{-17} \mathrm{~A}\left(\sim 1 \times 10^{-12} \mathrm{~A} / \mathrm{cm}^{2}\right)$, were measured near zero bias, indicative of high quality junction and low etch-related damage on the mesa sidewalls. The reverse leakage current remained below $60 \mathrm{nA}$ up to a bias of $-20 \mathrm{~V}$. The leakage current scales with the area of the diode, not its perimeter, and the surface leakage component thus appears negligible. The differential series resistance of our diodes scales with the mesa area, from 45 to $50 \Omega$ in $350 \mu \mathrm{m}$ devices 


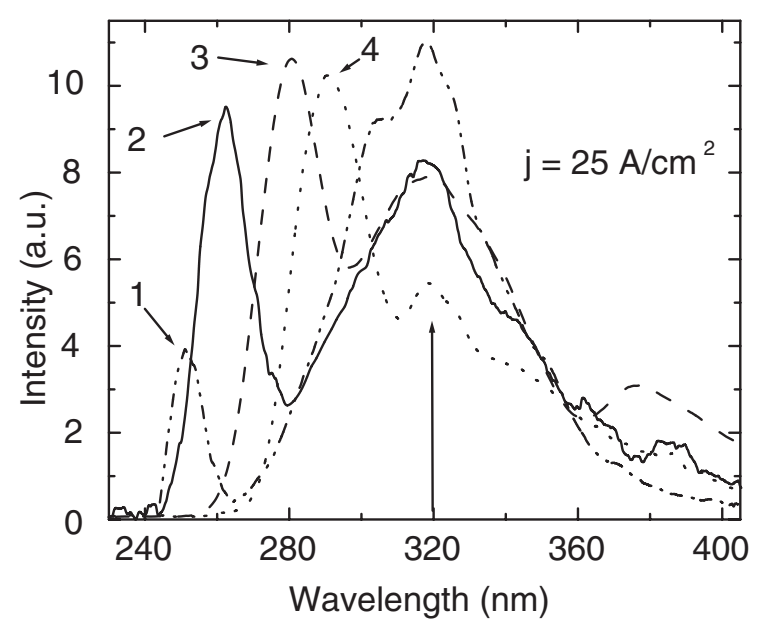

Fig. 9. Electroluminescence spectra of four LEDs operated at low current densities.

and from 110 to $130 \Omega$ in $110 \mu \mathrm{m}$ diodes.

EL spectra of LEDs operating at low dc current of $\sim 25$ $\mathrm{A} / \mathrm{cm}^{2}$ are shown in Fig. 9. LEDs operating at 250, 260, and $280 \mathrm{~nm}$ were grown on sapphire, with one operating at $290 \mathrm{~nm}$ grown on $\mathrm{Si}(111)$ substrate. Except for the LED grown on the Si substrate, all spectra were measured through the substrate. Two peaks are observed for each LED, one at the design wavelength, from 250 to $290 \mathrm{~nm}$, and a broad feature at around $320 \mathrm{~nm}$. From Fig. 9 we see that the intensities of the desired emission from LEDs operating at 260 to $290 \mathrm{~nm}$ are comparable. However, the LED operating at $250 \mathrm{~nm}$ has weaker emission intensity. This structure was produced using cladding layers which were the same as in our LEDs operating at longer wavelengths. Since the active region of the 250-nm LED has bandgap close to that of these cladding layers, we expect the carrier confinement to be poor. Improved emission intensity at $250 \mathrm{~nm}$ can be obtained by increasing the effective bandgap of the cladding layers $\left(x_{\mathrm{AlN}} \sim 0.78\right)$. N-type DAS with this composition are readily achievable. We are currently working on p-type DAS with the necessary compositions.

For spectra obtained with low drive current $\left(25 \mathrm{~A} / \mathrm{cm}^{2}\right)$, the broad emission competes with the desired LED output. As current is increased, the latter emissions grow rapidly in intensity while the former saturates. ${ }^{1,6)}$ The spectrum of the $280 \mathrm{~nm}$ LED operated at current density of $250 \mathrm{~A} / \mathrm{cm}^{2}$ is shown in Fig. 10. This spectrum is plotted on a semi-log scale in order to emphasize the weaker features. Under these conditions, the primary LED emission at $280 \mathrm{~nm}$ swamps out the $320 \mathrm{~nm}$ band by two orders of magnitude. The maximum output power of $160 \mu \mathrm{W}$ at $280 \mathrm{~nm}$ was measured under pulsed drive at a current density of $250 \mathrm{~A} / \mathrm{cm}^{2}$. The weak feature at $\sim 360 \mathrm{~nm}$ corresponds to luminescence from the contact GaN layer grown on the top of the LED.

A feature at $320 \mathrm{~nm}$ has been observed in $285 \mathrm{~nm}^{25)}$ and $292 \mathrm{~nm} \mathrm{LEDs}^{5)}$ based on $\mathrm{Al}_{x} \mathrm{Ga}_{1-x} \mathrm{~N}$ alloys. It was attributed to a free-carrier to deep acceptor transition in $\mathrm{Mg}$-doped $\mathrm{p}$ $\mathrm{Al}_{0.4} \mathrm{Ga}_{0.6} \mathrm{~N}^{5,25)}$ Such a transition could arise in our DASbased LED because of the formation of $1 \mathrm{ML}$ thick barrier/ well interfaces in which the AlN concentration may vary. The total volume fraction of these regions throughout the DAS can be considerable since the period is small, i.e., it

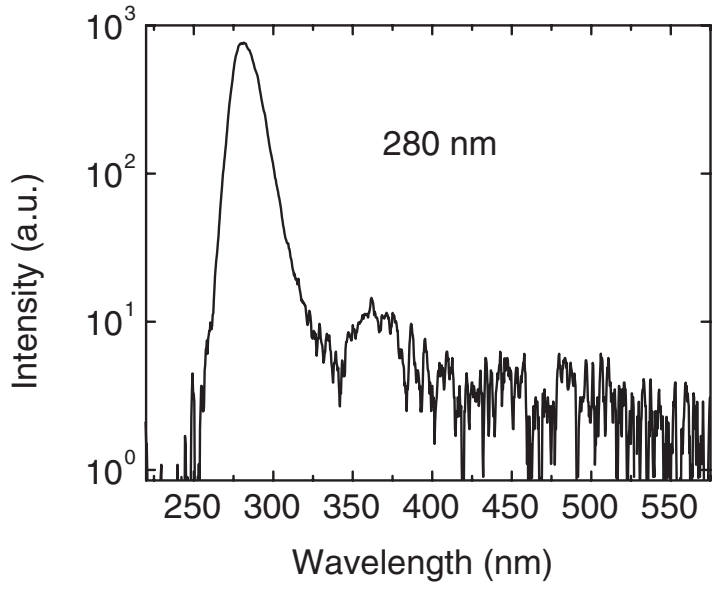

Fig. 10. Electroluminescence spectrum of a 280-nm LED operated at $250 \mathrm{~A} / \mathrm{cm}^{2}$.

scales like $\Delta L / L$, where $\Delta L$ is the effect of monolayer fluctuations and $L$ is the period. The positions of the $320 \mathrm{~nm}$ peak in Fig. 9 does not scale with the effective bandgap of the active region, making it difficult to assign our feature to a free-carrier to deep acceptor transition. Our CL studies, reported elsewhere, ${ }^{4)}$ show that the intensity of the long wavelength peak scales with Si doping in the n-type DAS. Additional experiments are required to pin down the origin of the $320 \mathrm{~nm}$ peak present in UV LEDs.

\section{Conclusions}

We have demonstrated $\mathrm{AlN} / \mathrm{Al}_{0.08} \mathrm{Ga}_{0.92} \mathrm{~N}$ p- and n-type DAS with high carrier concentrations. In particular, for DAS with average $x_{\mathrm{AIN}}$ of 0.72 , bandgap $5.1 \mathrm{eV}$, we obtain hole concentrations up to $10^{18} \mathrm{~cm}^{-3}$. We systematically studied the relationship between DAS architecture and energy gap, up to optical gaps of $5.3 \mathrm{eV}$. Based on these results, we produced LEDs with emission wavelengths between 290 and $250 \mathrm{~nm}$. Future work will focus on the development of $\mathrm{p}$ type DAS for cladding layers with higher average $x_{\mathrm{AIN}}$ up to 0.8 , corresponding to bandgaps of $\sim 5.2 \mathrm{eV}$.

\section{Acknowledgments}

This work is supported by DARPA (under a contract monitored by Dr. J. Carrano), NSF (ECS-0323640 and ECS0304224), RDECOM-US Army, NATO Science for Peace (974505), and the J. F Maddox Foundation. We also thank Dr. G. Kipshidze and Mr. B. Borisov for growth of the samples, Mr. V. Kuryatkov for device preparation and electrical measurements, Mr. A. Chandolu for XRD, Mr. I. Ahmad for optical measurements, and Dr. S. N. G. Chu for TEM measurements.

1) G. Kipshidze, V. Kuryatkov, B. Borisov, S. Nikishin, M. Holtz, S. N. G. Chu and H. Temkin: Phys. Status Solidi A 192 (2002) 286.

2) V. Adivarahan, J. Zhang, A. Chitnis, W. Shuai, J. Sun, R. Pachipulusu, M. Shatalov and M. A. Khan: Jpn. J. Appl. Phys. 41 (2002) L435.

3) A. Yasan, R. McClintock, K. Mayers, S. R. Darvish, P. Kung and M. Razeghi: Appl. Phys. Lett. 81 (2002) 801.

4) G. Kipshidze, V. Kuryatkov, K. Zhu, B. Borisov, M. Holtz, S. Nikishin and H. Temkin: J. Appl. Phys. 93 (2003) 1363.

5) A. Hanlon, P. M. Pattison, J. F. Kaeding, R. Sharma, P. Fini and S. Nakamura: Jpn. J. Appl. Phys. 42 (2003) L628. 
6) S. A. Nikishin, V. Kuryatkov, A. Chandolu, B. Borisov, G. Kipshidze, H. Temkin, I. Ahmad and M. Holtz: Jpn. J. Appl. Phys. 42 (2003) L1362.

7) A. J. Fischer, A. A. Allerman, M. H. Crawford, K. H. A. Bogart, S. R. Lee, R. J. Kaplar, W. W. Chow, S. R. Kurtz, K. W. Fullmer and J. J. Figiel: Appl. Phys. Lett. 84 (2004) 3394.

8) J. L. Darby, D. P. Y. Chang, P. S. Coggin, H. K. Chung and P. K. Dasgupta: Process Control Qual. 6 (1995) 229.

9) H.-K. Chung, H. S. Bellamy and P. K. Dasgupta: Talanta 39 (1992) 593.

10) P. K. Dasgupta, Q. Li, H. Temkin, M. H. Crawford, A. J. Fischer, A. A. Allerman, K. H. A. Bogart and S. R. Lee: Proc. SPIE 5530 (2004) 174.

11) Q. Li, P. K. Dasgupta, H. Temkin, M. H. Crawford, A. J. Fischer, A. A. Allerman, K. H. A. Bogart and S. R. Lee: Appl. Spectrosc. 58 (2004) 1360

12) G. Kipshidze, V. Kuryatkov, B. Borisov, M. Holtz, S. A. Nikishin and H. Temkin: Appl. Phys. Lett. 80 (2002) 3682.

13) H. Hirayama, A. Kinoshita, M. Ainoya, A. Hirata and Y. Aoyagi: Phys. Status Solidi A 188 (2001) 83.

14) Z. Sitar, M. J. Paisley, B. Yan, J. Ruan, W. J. Choyke and R. F. Davis: J. Vac. Sci. Technol. B 8 (1990) 316.

15) M. A. Khan, J. N. Kuznia, D. T. Olson, T. George and W. T. Pike: Appl. Phys. Lett. 63 (1993) 3470.

16) V. Kuryatkov, K. Zhu, B. Borisov, A. Chandolu, I. Gheriasou, G.
Kipshidze, S. N. G. Chu, M. Holtz, Yu. Kudryavtsev, R. Asomoza, S. A. Nikishin and H. Temkin: Appl. Phys. Lett. 83 (2003) 1319.

17) K. Zhu, V. Kuryatkov, B. Borisov, G. Kipshidze, S. A. Nikishin, H. Temkin and M. Holtz: Appl. Phys. Lett. 81 (2002) 4688.

18) T. V. Shubina, V. N. Jmerik, M. G. Tkachman, V. A. Vekshin, V. V. Ratnikov, A. A. Toropov, A. A. Sitnikova, S. V. Ivanov, J. P. Bergman, F. Karlsson, P. Holtz and B. Monemar: Phys. Status Solidi B 234 (2002) 919.

19) P. J. Schuck, M. D. Mason, R. D. Grober, O. Ambacher, A. P. Lima, C. Miskys, R. Dimitrov and M. Stutzmann: Appl. Phys. Lett. 79 (2001) 952.

20) V. Fiorentini: Appl. Phys. Lett. 82 (2003) 1182.

21) A. Chandolu, S. A. Nikishin and H. Temkin: unpublished.

22) M. Holtz, T. Prokofyeva, M. Seon, K. Copeland, J. Vanbuskirk, S. Williams, S. Nikishin, V. Tretyakov and H. Temkin: J. Appl. Phys. 89 (2001) 7977.

23) K. Zhu, V. Kuryatkov, B. Borisov, G. Kipshidze, S. A. Nikishin, H. Temkin and M. Holtz: Appl. Phys. Lett. 81 (2002) 4688.

24) K. Zhu, V. Kuryatkov, B. Borisov, J. Yun, G. Kipshidze, S. A. Nikishin, H. Temkin, D. Aurongzeb and M. Holtz: J. Appl. Phys. 95 (2004) 4635.

25) M. Shatalov, A. Chitnis, V. Mandavilli, R. Pachipulusu, J. P. Zhang, V. Adivarahan, S. Wu, G. Simin, M. A. Khan, G. Tamulaitis, A. Sereika, I. Yilmaz, M. S. Shur and R. Gaska: Appl. Phys. Lett. 82 (2003) 167 